\title{
INFLUÊNCIA DA RAÇA, SEXO E PESO/IDADE SOBRE O RENDIMENTO DA CARCAÇA EM CORDEIROS
}

\author{
THE INFLUENCE OF BREED, SEX AND WEIGHT/AGE ON CARCASS YIELD IN LAMBS
}

\author{
Maria Teresa Moreira Osório $^{1}$ Isidro Sierra $^{2}$ Carlos Sañudo $^{2}$ José Carlos Osório $^{3}$
}

RESUMO

O estudo objetivou quantificar e comparar o rendimento da carcaça em cordeiros machos e fêmeas, abatidos com 63 73 dias de idade (peso de carcaça fria entre 6,5 kg e 7,9 kg) e 80-90 dias de idade (peso de carcaça fria entre $8,0 \mathrm{~kg}$ e 9,5 kg), procedentes das raças Rasa Aragonesa, Ojinegra de Teruel e Roya Bilbilitana. Não foi verificada diferença de rendimentos de carcaça entre as raças; por outro lado, as fêmeas apresentaram rendimentos superiores aos machos; assim como os cordeiros de maior idade/peso de carcaça apresentaram rendimentos superiores aos de menor idadelpeso de carcaça. As raças que participam da denominação específica de qualidade "Ternasco de Aragón” (Rasa Aragonesa, Ojinegra de Teruel e Roya Bilbilitana) apresentam rendimentos de carcaca semelhantes. Entretanto, o sexo e a idadelpeso de carcaça são fatores de variação do rendimento de carcaça que devem ser considerados para uma uniformização e comercialização de um produto de qualidade.

Palavras-chave: ovino, cordeiro, rendimento de carcaça.

\section{SUMMARY}

This study aimed to compare and quantify carcass yield of male and female lambs of the following breeds: Rasa Aragonesa, Ojinegra de Teruel and Roya Bilbilitana. Animals were slaughtered, one group at 63-73 days of age (cool carcass weight between 6.5 and $7.3 \mathrm{~kg}$ ) and another when 80-90 days of age (cool carcass weight between 8.0 and $9.5 \mathrm{~kg}$ ). Carcass yield has not been influenced by breed. On the other hand, females have shown yields higher than the males; as well as lambs of higher age/weight of carcass did show higher yields than those of lower age/weight of carcass. These breeds, which comprise the ones that receive the specific denomination for quality "Ternasco de Aragón”, show similar carcass yields. However, sex and age/weight of carcass are variation factors of carcass yields which should be considered for a standardization and selling of a quality product.

Key words: sheep, lamb, carcass yield.

\section{INTRODUÇÃO}

O rendimento de carcaça é uma característica diretamente relacionada à produção de carne e pode variar de acordo com fatores intrínsecos e/ou extrínsecos ao animal; dentre esses fatores, encontramse a base genética, o sexo, o peso e a idade do cordeiro, alimentação, tipo de jejum e transporte (SAÑUDO \& SIERRA, 1993; SAÑUDO $\boldsymbol{e t}$ al., 1994; SIERRA $\boldsymbol{e t}$ al., 1994 e OSÓRIO et al., 1996). Quanto à raça, observa-se que os animais procedentes de base genética especializada para a produção de carne apresentam rendimentos de carcaça superiores (OSIKOWSKI \& BORYS, 1976; BONIFACINO et al., 1979; FIGUEIRÓ, 1979; OSÓRIO et al., 1991 e 1996). Por sua vez, a idade, que é um fator ligado ao peso e ao estado de engorduramento, apresenta uma expressiva influência sobre o rendimento da carcaça. Sendo que a uma maior idade, principalmente até a maturidade do cordeiro, há um superior peso vivo e de carcaça e, conseqüentemente, a partir de um momento determinado, rendimentos de carcaça superiores (LARGE, 1964; SOLOMON et al., 1980; SIERRA, 1981). No que se refere ao sexo, as fêmeas apresentam um maior rendimento de carcaça que os machos (BRADFORD $\&$ SPURLOCK, 1964); isso se deve a maior precocidade das fêmeas e, portanto, superior engorduramento (SIERRA, 1973; SAÑUDO, 1977). Portanto, buscar a idade e/ou peso de sacrifício em que o rendimento de carcaça seja economicamente o mais indicado, para machos e fêmeas em determinada raça ou grupos genéticos, é de grande valia para otimização dos sistemas de produção e, na comercialização dos animais para abate.

\footnotetext{
${ }^{1}$ Médico Veterinário, PhD, Professor Adjunto, Universidade Federal de Pelotas (UFPel), Faculdade de Agronomia Eliseu Maciel (FAEM), Zootecnia, 96010-900, Pelotas, RS. E-mail: mtosorio@minerva.ufpel.tche.br. Autor para correspondência.

${ }^{2}$ Médico Veterinário, PhD, Professor Catedrático, Universidad de Zaragoza, Facultad de Veterinária. España.

${ }^{3}$ Médico Veterinário, PhD, Professor Titular, UFPEL, FAEM, Zootecnia, Bolsista do CNPq. 
No caso da denominação específica "Ternasco de Aragón", o peso de carcaça fria é fixado entre 7,0 e 12,0kg; entretanto, buscando sistematicamente um produto de qualidade, com as duas idéias básicas de juventude e maciez, a opção de produzir carcaças leves (dentro do limite inferior da amplitude acima citada) é uma alternativa economicamente viável para os criadores e propiciará um produto mais uniforme a um consumidor cada vez mais exigente. $\mathrm{O}$ objetivo deste estudo foi quantificar e comparar o rendimento de carcaça de cordeiros machos e fêmeas, em duas idades de abate, nas raças da denominação específica de qualidade "Ternasco de Aragon" (Rasa Aragonesa, Ojinegra de Teruel e Roya Bilbilitana).

\section{MATERIAL E MÉTODOS}

Foram sacrificados 98 cordeiros, desmamados com 45-55 dias e submetidos a um sistema intensivo em confinamento, com alimentação de concentrado à vontade e palha de cereal até o abate (63-73 dias e 80-90 dias). A idade de 63-73 dias para abate foi fixada para obtenção de carcaça com peso frio entre $6,5 \mathrm{~kg}$ e $7,9 \mathrm{~kg}$ e a idade de abate de $80-90$ dias foi para obtenção de carcaça fria com peso entre $8,0 \mathrm{~kg}$ e $9,0 \mathrm{~kg}$. Destes cordeiros, 39 pertencentes à raça Rasa Aragonesa (19 machos e 20 fêmeas), 33 à raça Ojinegra de Teruel (14 machos e 19 fêmeas) e 26 à raça Roya Bilbilitana (12 machos e 14 fêmeas). Afora os fatores acima citados, as demais condições de criação dos cordeiros foram as mesmas, determinadas para a denominação específica de qualidade "Ternasco de Aragón” (SIERRA, 1974, 1986, 1988).

O peso vivo foi tomado imediatamente antes dos animais serem abatidos, com um jejum de 18 horas. Posteriormente, os cordeiros foram abatidos, tomado o peso de carcaça quente, imediatamente ao abate, e colocadas as carcaças em câmara fria, a $1^{\circ} \mathrm{C}$ por 14 horas; logo, foram retiradas e pesadas, peso de carcaça fria. A partir do peso vivo ao sacrifício (PVA), peso de carcaça quente (PCQ) e peso de carcaça fria (PCF) foram calculados o rendimento verdadeiro [(PCQ/PVA).100] e o rendimento comercial [(PCF/PVA).100]. Através da análise de variância dos dados, foi verificado o efeito da raça, sexo e peso/idade de abate sobre o rendimento de carcaça verdadeiro e comercial. O modelo matemático utilizado foi $\mathrm{Y}=$ média + raça $\mathrm{i}+\operatorname{sexo} \mathrm{j}+$ peso/idade $1+$ interações $\mathrm{ij} 1+$ erro experimental ijlm; onde, $\mathrm{Y}$ é o rendimento de carcaça verdadeiro e comercial.

\section{RESULTADOS E DISCUSSÃO}

O efeito raça não foi significativo sobre os rendimentos de carcaça e, igualmente, observa-se que as médias não mostram uma diferença ou tendência que possa ter importância prática (Tabela 1). Isto, certamente, deve-se ao fato de que essas raças são de similar morfologia; como foi observado por OSÓRIO et al. (1996), das cinco raças estudadas, três não diferiam entre si e mostravam rendimentos de carcaça significativamente distintos das outras duas.

Verifica-se na Tabela 2, que as fêmeas apresentaram rendimentos de carcaça superiores aos machos, como era esperado, devido a maior precocidade das fêmeas e, conseqüentemente, seu superior estado de engorduramento (OSÓRIO, 1996), como já foi colocado em evidência nos estudos realizados por SAÑUDO (1980) e SIERRA (1980, 1983). Foi observado um efeito altamente significativo da idade/peso sobre os rendimentos de carcaça (Tabela 3); portanto, a uma maior idade, maior peso de carcaça e, conseqüentemente, superiores rendimentos de carcaça (SIERRA, 1973 e 1981; SOLOMON et al., 1980; CAÑEQUE et $\boldsymbol{a l} .$, 1996).

Igualmente, dentro da raça Rasa Aragonesa, foi observado um efeito significativo da idade/peso sobre os rendimentos de carcaça (verdadeiro e comercial); porém, nas outras duas raças (Ojinegra de Teruel y Roya Bilbilitana) não foi significativa a diferença sobre os rendimentos de carcaça verdadeiro, e isso se deve ao menor grau de liberdade (número de repetições) e maior variabilidade nos rendimentos verdadeiro dessas raças. Mas, igualmente, nessas raças, os cordeiros de maior idade apresentaram superiores rendimentos.

\section{CONCLUSÕES}

As raças que participam da denominação específica de qualidade "Ternasco de Aragón", apresentam rendimentos de carcaça verdadeiro e comercial semelhantes que variam, respectivamente, entre
Tabela 1 - Médias e erros-padrões, em \%, do rendimento de carcaça verdadeiro (RV) e comercial (RC) segundo raça.

F-TEST R. ARAGONESA $n=39$ OJINEGRA T. $n=39 \quad$ ROYA B. $n=26$

\begin{tabular}{lllll}
\hline & & & & \\
RV & n.s. & $48,77 \pm 0,35$ & $48,24 \pm 0,36$ & $48,09 \pm 0,43$ \\
RC & n.s. & $47,62 \pm 0,29$ & $47,03 \pm 0,30$ & $47,07 \pm 0,36$
\end{tabular}

n.s. $=(\mathrm{p}>0,05)$ 


\begin{tabular}{|c|c|c|c|}
\hline & F-TEST & MACHOS & FÊMEAS \\
\hline RV & * & $47,84 \pm 0,33$ & $48,89 \pm 0,30$ \\
\hline $\mathrm{RC}$ & n.s. & $46,90 \pm 0,27$ & $47,58 \pm 0,25$ \\
\hline
\end{tabular}

Centro de Ciências Rurais, Santa Maria, RS, v. 9, p. 421-428, 1979.

LARGE, R.V. The development of the lamb with particular reference to the alimentary tract. Animal Producción, v. 6, p. 169-178p, 1964.

OSIKOWSKI, M., BORYS, B. Effect on production and carcass quality characteristics of wether lambs of crossing Blackheaded Mutton, Ile de France and Texel rams with Polish Merino ewes. Livestock Production Science, v. 3, p. 343-349, 1976.

OSÓRIO, J.C.S., SIERRA, I., SAÑUDO, C., et al. Componentes do peso vivo em cordeiros e borregos Polwarth e cruzas Texel $\mathrm{x}$ Polwarth. In: ANAIS DO CONGRESSO INTERNACIONAL DE ZOOTECNIA. Évora, Portugal, Anais... 1991, p. 49$50,1991$.

Tabela 3 - Médias e erros-padrões, em \%, do rendimento de carcaça verdadeiro (RV) e comercial (RC), segundo idade.

\begin{tabular}{cccc}
\hline & & & \\
& F-TEST & 63-73 DIAS $\mathrm{n}=49$ & $80-90$ DIAS $\mathrm{n}=49$ \\
RV & $* *$ & $47,77 \pm 0,30$ & $48,97 \pm 0,33$ \\
RC & $* *$ & $46,39 \pm 0,25$ & $48,09 \pm 0,27$ \\
R. ARAGONESA & & $\mathrm{n}=15$ & $\mathrm{n}=24$ \\
RV & $*$ & $48,04 \pm 0,48$ & $49,51 \pm 0,36$ \\
RC & $*$ & $46,78 \pm 0,50$ & $48,45 \pm 0,38$ \\
O. TERUEL & & $\mathrm{n}=16$ & $\mathrm{n}=17$ \\
RV & n.s. & $47,65 \pm 0,62$ & $48,84 \pm 0,63$ \\
RC & $* *$ & $46,02 \pm 0,40$ & $48,04 \pm 0,41$ \\
R. BILBILITANA & & $\mathrm{n}=18$ & $\mathrm{n}=08$ \\
RV & n.s. & $47,63 \pm 0,42$ & $48,55 \pm 0,62$ \\
RC & $*$ & $46,37 \pm 0,38$ & $47,77 \pm 0,56$ \\
& & & \\
$* *$ P $<0,01)$ & $*=(\mathrm{P}<0,05)$ & n.s. $=(\mathrm{P}>0,05)$ &
\end{tabular}

$48,09 \%$ e $48,77 \%$ e entre $47,03 \%$ e $47,62 \%$. O sexo e a idade/peso de sacrifício são fatores de variação dos rendimentos de carcaça que devem ser considerados, quando se busca uma uniformização e comercialização justa de um produto de qualidade.

\section{REFERÊNCIAS BIBLIOGRÁFICAS}

BONIFACINO, L., KREMER, R., ORLANDO, D., et al. Estudio comparativo de corderos Corriedale y Corriedale por Texel. II. Pesos al nascer, ganâncias diárias y características de la canal. Veterinária, Montevideo, v. 70, p. 63-71, 1979.

BRADFORD, G.E., SPURLOCK, G.M. Effect of castrating lambs on growth and body composition. Animimal Producción, v. 6, p. 291, 1964.

CAÑEQUE MARTINEZ, V., LAUZURICA, S., LÓPEZ, D., $\boldsymbol{e}$ al. Producción de carne en corderos lechales de raza Talaverana. I. Rendimientos en el matadero e importancia de los despojos. In: XXI ${ }^{\mathrm{a}}$ JORNADAS CIENTIFICAS DE LA SOCIEDAD ESPAÑOLA DE OVINOTECNIA Y CAPRINOTECNIA. Logroño-Espanha, 1996. Anales... S/n.

FIGUEIRO, P.R.P. Efeito do cruzamento da raça Hampshire Down e Romney Marsh na produçao de cordeiros para abate. Revista do
OSÓRIO, J.C., OLIVEIRA, N.M., MONTEIRO, E., et al. Producción de carne en ovinos de cinco genotipos en Brasil. In: XXI ${ }^{\mathrm{a}}$ JORNADAS CIENTIFICAS DE LA SOCIEDAD ESPAÑOLA DE OVINOTECNIA Y CAPRINOTECNIA. Logroño - Espanha, 1996. Anales... S/n.

OSÓRIO, M.T.M. Estudio comparativo de la calidad de la canal y de la carne en las razas Rasa Aragonesa, Roya Bilbilitana y Ojinegra de Teruel. 299p. Tesis (Doctoral) - Facultad de Veterinaria. Universidad de Zaragoza. Zaragoza, España, 1996.

SAÑUDO, C. Influencia del sexo en el rendimiento canal en la especie ovina. In: II $^{\mathrm{a}}$ JORNADAS DE OVINOTECNIA. Anales... Mérida, España, p. 229-240, 1977.

SAÑUDO, C. Calidad de la canal y de la carne en el ternasco aragonés. 337p. Tesis (Doctoral) - Facultad de Veterinaria. Universidad de Zaragoza. Zaragoza, España, 1980.

SAÑUDO, C., SIERRA, I. Calidad de la canal y de la carne en la especie ovina. Ovino y Caprino. Monografias del Consejo General de Colegios Veterinarios. Madrid. España, p. 207-254. 1993.

SAÑUDO, C., SIERRA, I., OLLETA, J.L., et $\boldsymbol{a l}$. Influencia del destete en la calidad de la canal y de la carne en Ternasco de Aragón. In: XIX JORNADAS CIENTIFICAS DE LA SOCIEDAD ESPAÑOLA DE OVINOTECNIA Y CAPRINOTECNIA. Burgos-Espanha, 1994. Anales... p. 76-81.

SIERRA, I. Producción de cordero joven y pesado en la raza Rasa Aragonesa. I.E.P.G.E., (Zaragoza-Espanha), n.18, 28 páginas, 1973.

SIERRA, I. El "ternasco" aragones: descripción y caracteristicas fundamentales. Instituto de Economia y Producciones Ganaderas del Ebro (I.E.P.G.E.), Universidad de Zaragoza. Zaragoza-Espanha, n. 7, 9 páginas, 1974.

SIERRA, I. Résultats du croisement industriel de béliers Fleischschaf et Suffolk avec des brebis Rasa Aragonesa. $1^{\mathrm{a}}$ Consult. FAO, CEE, CIHEAM sur l'amelioration de génétique des ovins en Mediterranée, 2-4 Diciembre 1980. Zaragoza, 14 páginas, 1980.

SIERRA, I. Résultats du croisement industriel de béliers Fleischschaf Méditerranéennes. Serie Etudes. Le Croisement Industriel Ovin en Méditerranée. Instituto Agronomico Mediterraneo de Zaragoza. (Zaragoza-Espanha), p. 83-92, 1981. et Suffolk avec des brebis Rasa Aragonesa. Options 
SIERRA, I. Growth rate and other parameters in lambs: Effects of genotipe, sex, type of birth and lambing season. In: $34^{\text {th }}$. AN MEETING EAAP, Madrid, II, 1983. Anais... p. 586-587.

SIERRA, I. La denominación de origem en el ternasco de Aragón. Información Técnica Economica Agraria, (ZaragozaEspanha), n. 66, p. 3-12, 1986.

SIERRA, I. La denominación de origen en el ternasco de Aragón. SURCOS, (Revista Técnica del Departamento de Agricultura, Ganaderia y Montes de la Diputación General de Aragón. Zaragoza-Espanha, marzo 1988, n. 5, p. 27-29, 1988.
SIERRA, I., SAÑUDO, C., OLLETA, J.L., et al. Calidad de la canal y de la carne en el lechazo de Castilla. I. Efecto de la base genetica. In: XIX JORNADAS CIENTIFICAS DE LA SOCIEDAD ESPAÑOLA DE OVINOTECNIA Y CAPRINOTECNIA. Burgos-Espanha, 1994. Anales... p. 66-70,

SOLOMON, M.B., KEMP, J.D., MOODY, W.G. et al. Effect of breed and slaughter weight on physical, chemical and organoleptic properties of lamb carcasses. J. of Anim. Science, v. 51, n. 2, p. 1102-1107, 1980.

Ciência Rural, v. 29, n. 1, 1999. 